\title{
POSSIBILITIES OF EXPERIMENTAL VERIFICATION OF THE VALIDITY OF URETHRAL PRESSURE PROFILOMETRY
}

\author{
Frantisek Zatura $^{\mathrm{a} *}$, Josef Rosenberg ${ }^{\mathrm{b}}$
}

\author{
${ }^{a}$ Faculty of Medicine and Dentistry, Palacky University, Olomouc \\ ${ }^{b}$ Department of Mechanical Engineering, University of West Bohemia, Pilsen \\ e-mail: zaturaf@tunw.upol.cz
}

Received: October 10, 2006; Accepted: November 11, 2006

Key words: Urethral pressure profile/Profilometry/Experimental measurement

Objectives: The aim is to define conditions for exact measurement of urethral pressure profile, to prepare an aparatus and fabricate an artificial urethra for testing measuring catethers. To examine it's qualities by experiment.

Methods: We designed a measuring appliance and a specialized software for measuring and evaluating. An experimental phantom of urethra, made from a part of bull's urethra, was used for testing of measurement's validity with use of different measuring catethers. Measurements were 10x repeated within the same position of the catether. Reproducibility of the measurement and stability of the experimental model was tested.

Results: Measuring urethra and experimental phantom of urethra succeeded, it was possible to make well reproducible measurements with different measuring catethers and to evaluate their metrologic abilities and limits. Results of our measurements confirmed appointed theoretical conditions of the measurement.

Conclusion: Measurements proved that it is possible to simulate the conditions that are similar to physiological conditions and that it is possible to test metrological qualities of compressive profilometry of urethra.

\section{INTRODUCTION}

Urethra pressure profilometry was suggested by Brown and Wickham in $1969^{1}$. This is relatively simple method for examining the condition of the urethral closure mechanism when the external sphincter of the urethra was stimulated by electrical stimulators for incontinency and replaced the unsatisfactory methods of the intraluminal balloon sensor. When defining a method, basic presumptions have been established for proper measurement ${ }^{1-4}$. They are as follows: an ideal contact of measuring the side openings of the measuring catheter and a distensible urethra which is closed in the idle state. Perfect contact between the measuring hole and the urethral wall is very important. Profilometry gradually became a standard examination for incontinency. As examinations showed poor reproducibility, various new types of measuring catheters have been developed not only for perfuse but also with microsensors.

Profilometry is currently used both for the evaluation of the urethral sphincter and defecation function. However, a generally accepted definition of urethral pressure is missing and the specification of the method is not generally accepted. Procedures recommended by the ICS were developed in 1988 and 1989, the last standardisation is from 2002 (ref. ${ }^{5}$ ). Urethral pressure is defined here as the pressure of a liquid needed to open a closed (collapsed) urethra. The easy option to convert to pressure measurement is an advantage; where catheters are equipped with a sensor, pressure is developed by direct pushing of the urethral wall against the microsensor.
Regarding the necessary interaction of the wall with the sensor, the measurement depends on the shape and stiffness of the catheter with significant dependence on the position of the measuring unit in the urethra.

\section{PHYSICAL BACKGROUND OF PROFILOMETRY}

During profilometry, liquid is expelled from the hole on the catheter surface with a constant flow rate of $Q$ $\left[\mathrm{m}^{3} / \mathrm{s}\right.$ resp. $\left.\mathrm{cm}^{3} / \mathrm{s}\right]$ and relevant pressure of $Q[P a$ resp. $\mathrm{cm} \mathrm{H}_{2} \mathrm{O}$ or $\left.\mathrm{mmHg}\right]$ is measured. The value of this pressure is supposed to be dependent on the properties of the urethral wall in the location of the hole. Let's perform a more detailed analysis of this assumption:

Two configurations of the catheter hole are assumed. (Fig. 1 and 2)

Fig. 1.

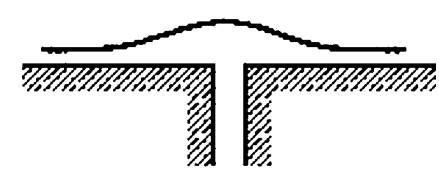

Fig. 2.

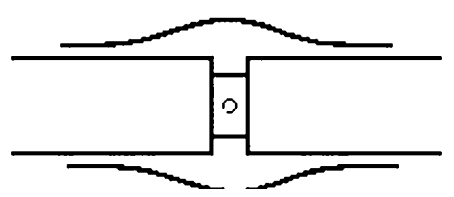


Figure 1 shows a hole located on the surface of the catheter. On Figure 2, the hole is at the bottom of the circumferential groove.

The first essential fact is that correlation between the pressure, $p$ and flexibility of the urethral wall in the location of circumference exists only if the urethra at zero pressure, $p$ directly fits close to the hole. Otherwise, there is a case of flow in a channel with a variable cross-section where the pressure and speed ratios are given by the boundary conditions at the input (pressure $p$ in the hole) and at its end. This, however cannot be defined - it means remote locations of the gap between the catheter and urethra and moreover, they have no relation to the property in the area of the hole.

Let's consider the configuration described in Fig. 1. Schematically, it is transferred into a scheme as shown in Fig. 3.

The common applied constitutive relation for the urethral wall is expressed as follows

$$
A=\phi(p),
$$

where $A\left[\mathrm{~m}^{2}\right]$ is the cross-section area. The following linear constitutive relation is often applied

$$
A=A_{0}+\beta p,
$$

where $A_{0}$ and $\beta$ are material parameters of the urethral wall that are changing along its length. $A_{0}$ is the cross-section area corresponding to the empty urethra (pressure equel zero). If the relations from Figure 2 are applied, the following relations can be given ( $\mathrm{F}$ is the catheter circumference ).

$$
\pi D=F \Rightarrow D=\frac{F}{\pi}
$$

$A=\frac{\pi}{4}(D+2 x)^{2}=\frac{\pi D^{2}}{4}+\pi D x+\pi x^{2} \doteq \frac{\pi D^{2}}{4}+\pi D x$.

Area $\tilde{A}$, through which the liquid is forced from the hole, is given as the following relation

$$
\tilde{A}=\pi d x .
$$

Now A shall be replaced with the relevant figures from (4) and (1), making

$$
\frac{\pi D^{2}}{4}+\pi D x=\phi(p) .
$$

From this equation, $\mathrm{x}$ can be calculated and put into (5)

$$
\tilde{A}=\pi d \frac{\phi(p)-\frac{\pi D^{2}}{4}}{\pi D}=\frac{d}{D}\left(\phi(p)-\frac{\pi D^{2}}{4}\right) .
$$

In the case when the urethral wall for $p=0$ fits close to the hole (i.e. $p=0 \Rightarrow x=0$ ), the flow rate depends only on

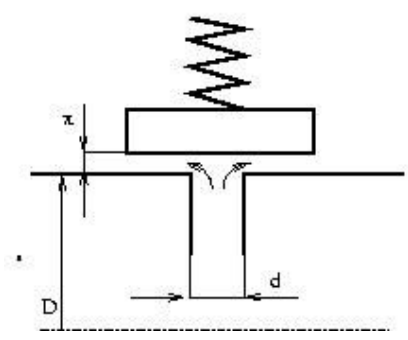

Fig. 3.

the input, i.e. p and Q (Note: This is called the "waterfall effect" - the flow speed at the beginning of the waterfall does not depend on its height). We can demonstrate that rate $u$ equals the critical rate $c$, i.e. the speed of pressure pulsation transmission, given by the following relation

$$
c=\sqrt{\frac{\tilde{A}}{\rho \frac{d \tilde{A}}{d p}}} \cdot
$$

For flow volume $\mathrm{Q}$, the following equation is relevant

$$
Q=c \tilde{A} \text {. }
$$

After replacing the corresponding figures from (7), we have the basic relation

$$
Q^{2}=\frac{\left[\frac{d}{D}\left(\phi(p)-\frac{\pi D^{2}}{4}\right)\right]^{3}}{\rho \frac{d}{D} \frac{d \phi}{d p}} .
$$

or

$$
\rho \frac{d}{D} \frac{d \phi}{d p} Q^{2}=\left(\frac{d}{D}\right)^{3}\left(\phi(p)-\frac{\pi D^{2}}{4}\right)^{3} .
$$

If a linear connection is applied (2), it transfers (10) into the following form

$$
\rho \frac{d}{D} \beta Q^{2}=\left(\frac{d}{D}\right)^{3}\left(A_{0}+\beta p-\frac{\pi D^{2}}{4}\right)^{3} .
$$

Case $1 \mathrm{~b}$ is different only in relation for $\tilde{A}$

$$
\tilde{A} \doteq 2 \pi D x .
$$

Having replaced $\mathrm{x}$ for (6), we receive

$$
\tilde{A}=2 \pi D \frac{\phi(p)-\frac{\pi D^{2}}{4}}{\pi D}=2\left(\phi(p)-\frac{\pi D^{2}}{4}\right)
$$

and from (9), the result is as follows 


$$
Q^{2}=8 \frac{\left(\phi(p)-\frac{\pi D^{2}}{4}\right)^{3}}{\rho \frac{d \phi}{d p}}
$$

or $\quad Q^{2} \rho \frac{d \phi}{d p}=8\left(\phi(p)-\frac{\pi D^{2}}{4}\right)^{3}$.

Thereafter, we receive the relation for (2)

$$
Q^{2} \rho \beta=8\left(A_{0}+\beta p-\frac{\pi D^{2}}{4}\right)^{3} .
$$

Regarding the basic condition that the urethra should fit close to the hole, configuration $1 \mathrm{~b}$ appears unsatisfactory. Due to the irregular shape of the urethral lumen, the urethra cannot fit along the entire circumference of a catheter.

The only possibility might be to place more holes on the catheter along its circumference where the wall should fit at least one hole. It shows the highest pressure therein and for the determination of material properties, relation (10) or (11) can be applied.

The findings mentioned above for measurement methods indicate that perfect contact of the measuring unit with the urethral wall is necessary, indeed. This can be achieved, for instance through the following procedures:

- to apply a catheter with a diameter close to the diameter of the urethra. As the urethral diameter is not equal along the entire length, the necessary conditions of measurement cannot be assured by this method in the full range. It is also questionable, what diameter size of catheter to apply to a urethra containing flexible elements and which in idle state is basically fully closed.

- The next possibility is to create a measurement system of more sensors distributed along the circumference of a catheter. If we accept the theory that ideal values are measured at the places of perfect contact with the urethral wall surface, there is the potential that at least one of these measuring elements on the cath-

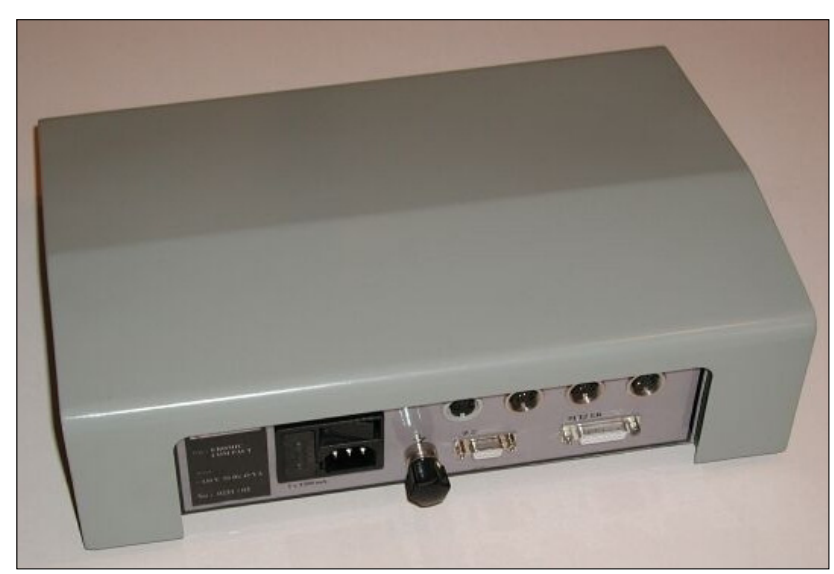

Fig. 4. eter circumference will always have perfect contact with the surface of the urethral wall. For this purpose, multi-channel catheters can be used (with 4,6 or 8 channels). We can further assume that the pressure in this ideal place will be the highest of all the measured values in one cross-section.

- A sensor with a miniaturized balloon and pressure transfer from the entire wall diameter through this balloon can be applied.

- A system with an electronic chip built in the wall of the measuring catheter (MikroTip) should also be considered.

It is impossible to compare and interpret the metrological properties of these systems due to the fact that identification of the material properties of the urethra is quite difficult to perform with the use of mathematical methods, and the use of a clinical test is restricted due to ethical reasons. Thus only experimental measurement is available, with the aim to approximate as much as possible the conditions of measurement in clinical practice.

\section{OBJECTIVE}

This work is aimed at developing experimental devices (apparatuses) for urethral pressure profilometry which could measure using all the methods as mentioned above.

The second task was to prepare a model of a urethra with sufficient stability so that its properties would not vary during repeated measurements.

The third task was to find methods of interpreting the evaluated parameters.

\section{MATERIAL AND METHODS}

The Uromic Compact (Condata Olomouc), a wellproven device with required certificates $(\mathrm{CB}, \mathrm{CE}$ certificates), has been modified to be used as an apparatus for these universal biometric measurements. The device was modified to a functionality, which encloses only the pressure channels and added control circuits for an accurate profilometric withdrawing unit (Fig. 3 and 4). The device is controlled by computer and all measurements were stored on the hard disk for later evaluation.

Specialized SW providing the option of free programming of measuring channels for various types of sensors and measurement methods has been developed. A module for the presentation of multi-channel measurement using vector graphs and a module which allows reading onechannel examinations one over another for an easy comparison analysis of individual curves has been designed as an original solution.

We executed a detailed calibration procedure of each particular channel, which was enclosed in the SW; a digital pressure gauge Greisinger GMH 3160-07B, officially calibrated in an accredited Meros laboratory, was used for calibration and control of long-term duration.

As our first measurements showed that the standard 


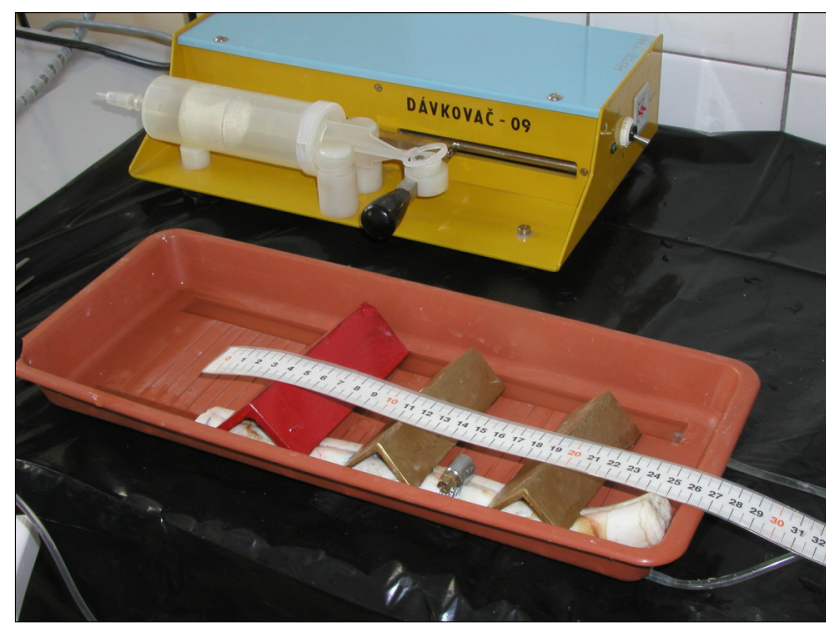

Fig. 5.

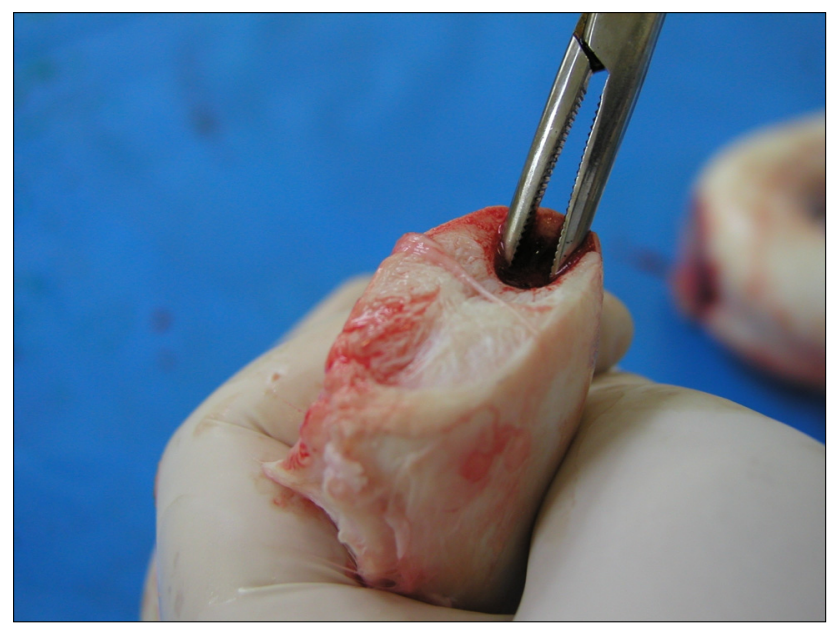

Fig. 6.

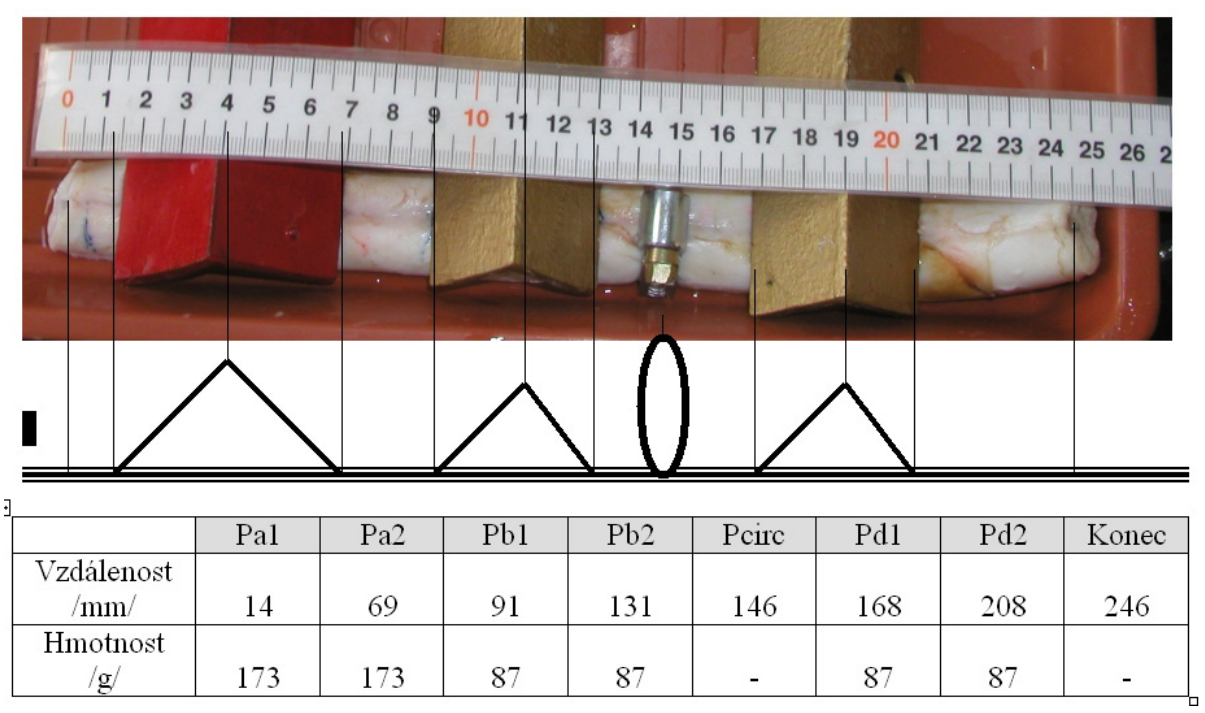

Fig. 7.

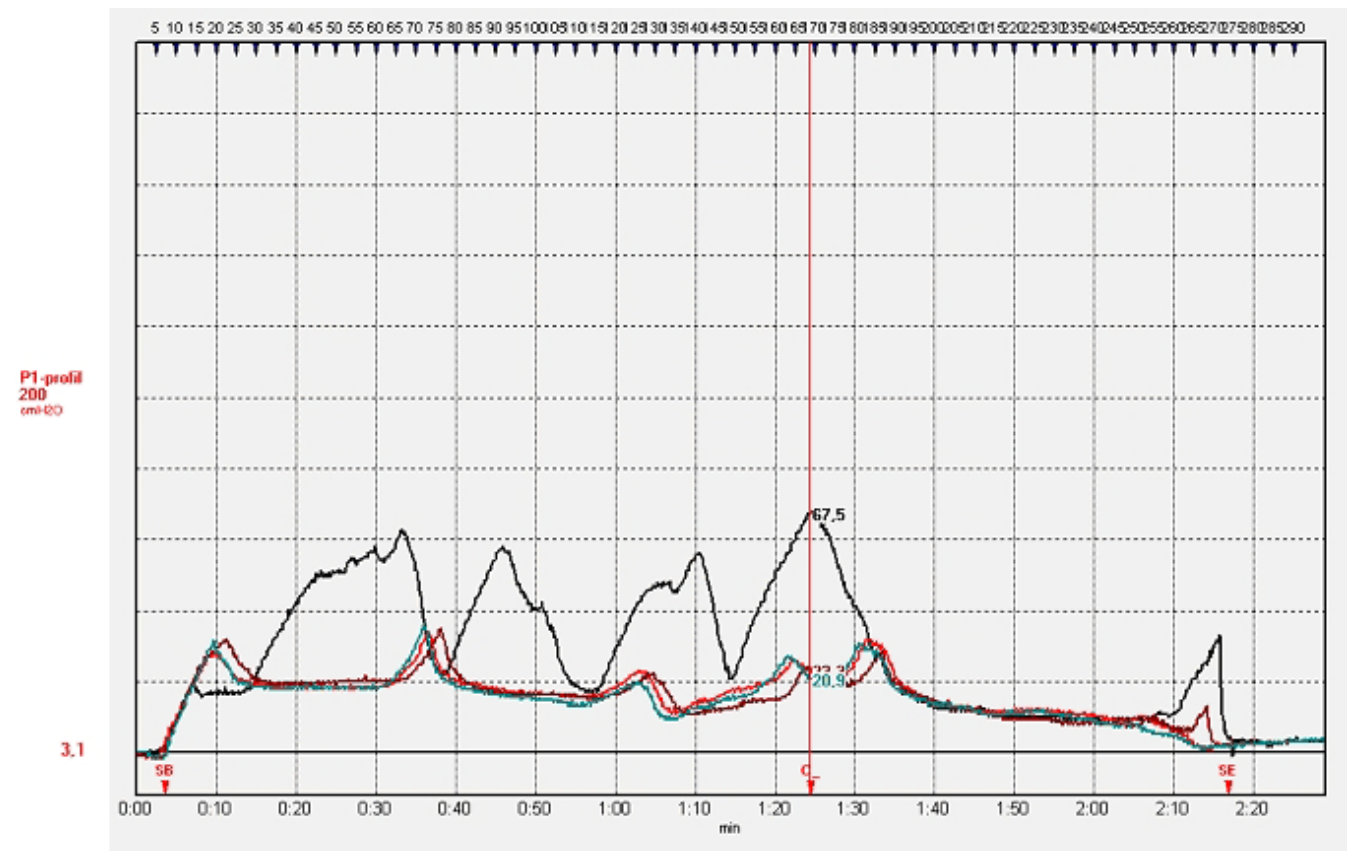

Fig. 8. 


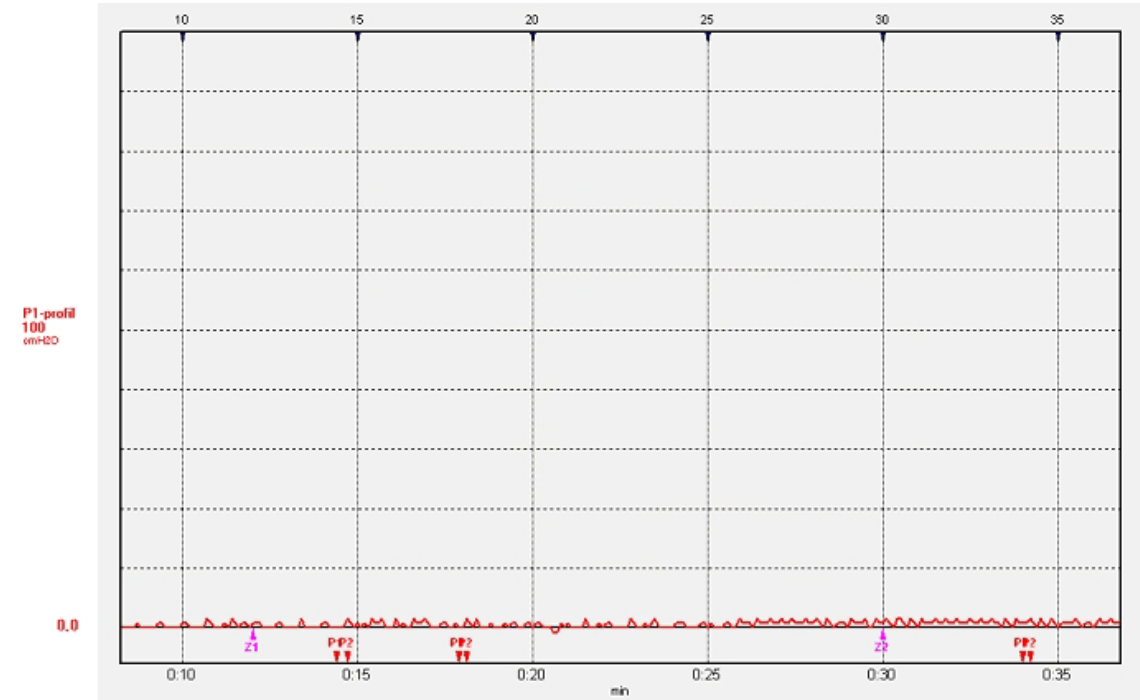

Fig. 9.

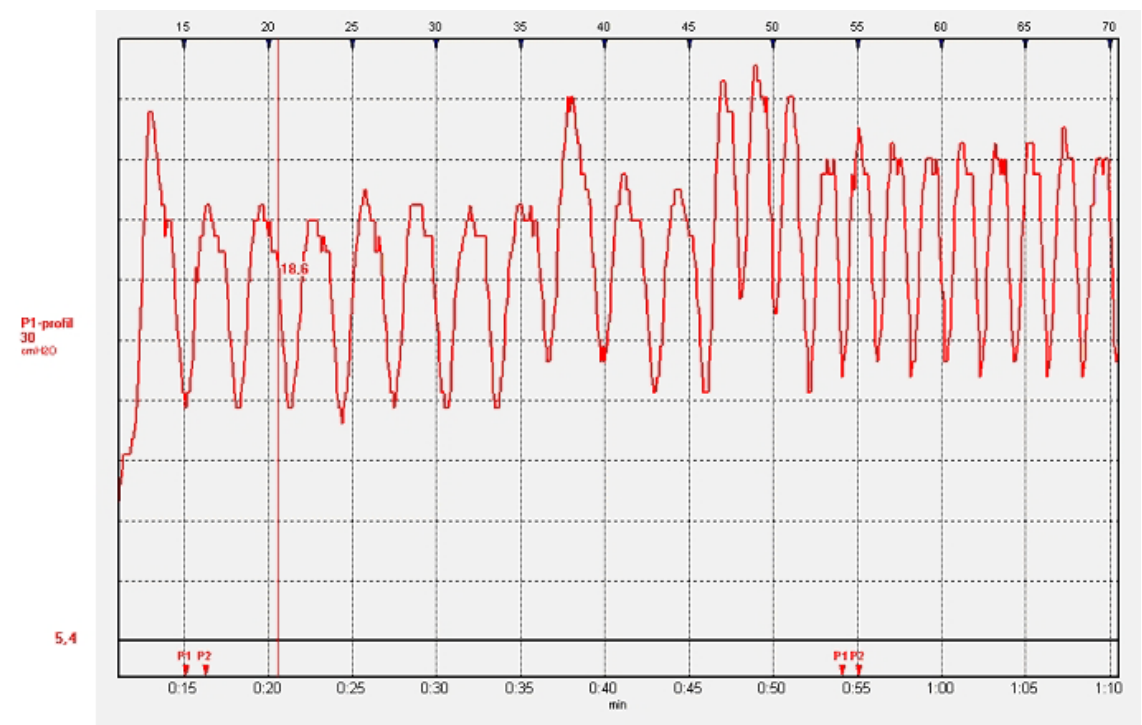

Fig. 10.

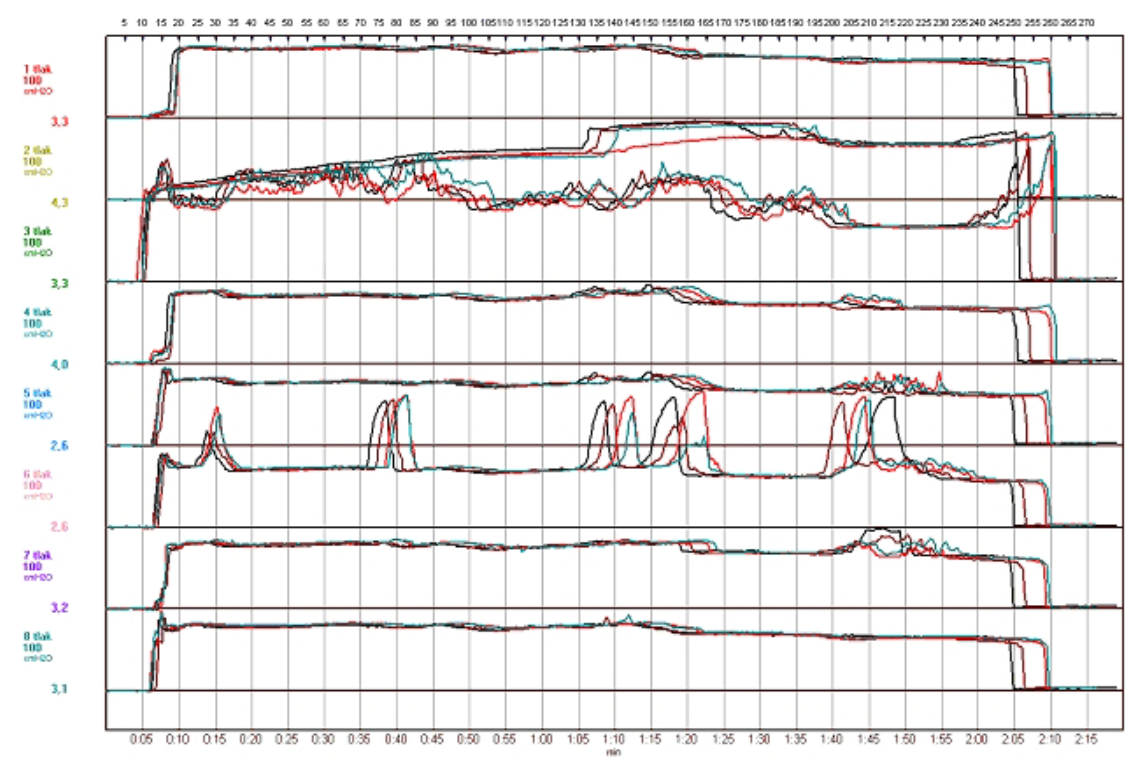

Fig. 11. 


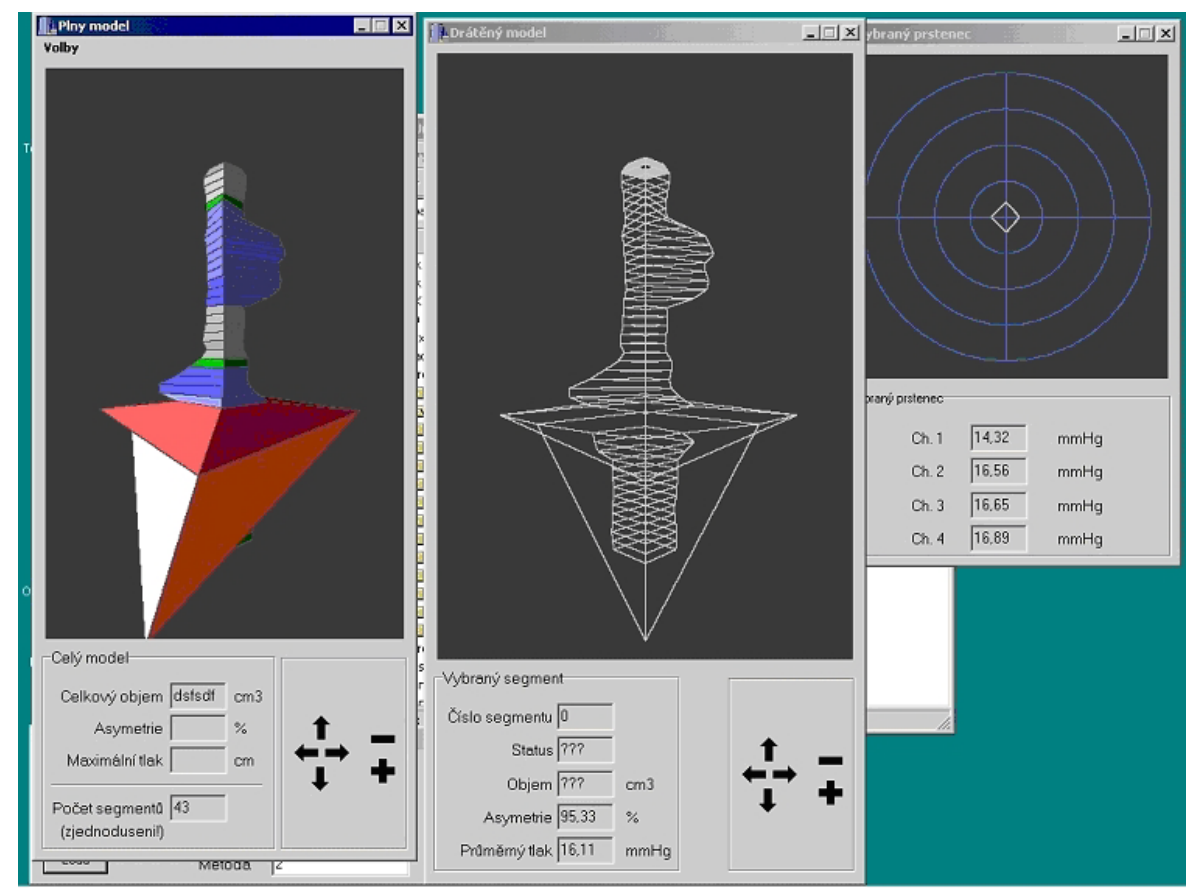

Fig. 12.

perfuse pumps of urodynamic devices caused an error in measurement in a pressure range of $1-3 \mathrm{~cm} \mathrm{H}_{2} \mathrm{O}$ (we used a linear pump with digital control of force movement [Trystom Olomouc], which was originally developed just for these purposes [Fig. 4]).

A standard 8-channel device, Uromic6 with the same SW and measurement method was used for 8-channel measurements.

It was quite difficult to prepare an experimental urethra. Constructions with silicon or latex tubes used by some authors failed due to the insufficient collapsing ability of these materials to provide perfect contact with the measuring hole. That's why we focused on animal organs. Regarding the required purpose and also due to ethical and practical reasons, we used only organs from dead animals after our first few experiences. Rat urethras ${ }^{3} \mathrm{did}$ not match in diameter, pig urethras, apart from the inappropriate diameter caused problems with the path of the urethra (sigma) which prevented easy introduction of a measuring catheter.

Finally, bull urethras proved to be the best solution, acquired from a slaughterhouse together with the penis of the animal immediately after it had been slaughtered. The preparation was transported with ice similarly to organs for transplantation. A $30-\mathrm{cm}$ piece of the urethra together with cavernous bodies (Fig. 5) was extracted; the diameter of the urethra was controlled by a calibration catheter 28-30 charr. The structure of the urethra is very similar to a human urethra (Fig. 6), the lumen of urethra collapses easy and adjoins well at the wall of the measuring catheter.

Changes of pressure against the urethral wall were simulated by attaching metal angles with a defined weight on the surface of the urethra, and by circular compression using a clip (Figs. 7 and 8).

\section{RESULTS}

The described original experimental set was used to test all the measuring catheters available on the Czech market, as well as other experimental catheters; we performed more than 300 individual measurements in total.

The long-term stability of the measurements was verified by a hydrostatic method. The pressure in a cylinder filled with water was at the same level after 8 hours, variations occurred of less than $1 \mathrm{~cm}$ of $\mathrm{H}_{2} \mathrm{O}$.

The properties of perfuse pumps have been evaluated at rates of 1,2 and $3 \mathrm{~cm}$. The original digitally controlled linear pump proved the most suitable for measurement, standard pumps of urodynamic equipment showed an overly high fluctuation of the flow rate, which was reflected in the artificial fluctuation of measured pressure in the urethra (Figs. 9 and 10)

The model of the urethra was assessed from the point of view of whether valid data can be measured by each particular method and whether the data are reproducible even after a longer time of using the model.

Using all measurement catheters, we obtained valuable signals; we were able to assess the sensitivity of measurement, measurements in each particular position with regard to the position of the measuring hole in the urethra diameter (marked clockwise and at an angle of $0^{\circ}-360^{\circ}$ )

The stability of the experimental model was evaluated by a comparison of 8-channel measurements at one-hour intervals, each time alternating with the next measurement. Regarding the fact that examinations were easy to reproduce even after an entire cycle of measurements (Fig. 11), we consider the bull urethra to be sufficiently stable and resistant for use as a urethra test model. Two new methods of graphic evaluation - the superposition of 
some curves of pressure progression (Fig. 11) and a vector graph for the evaluation of multi-channel measurements (Fig. 12) enabled a highly illustrative evaluation of more complicated measurements of biometric parameters for which the classic statistical method cannot be applied easily.

\section{DISCUSSION}

Issues concerning the metrological verification of profilometry are published quite rarely. For a validity study of profilometry, a mechanical urethral model ${ }^{6}$, urethral lumen from rabbit intestine and a urethra from a live female $\operatorname{dog}^{7}$ have been applied. These models, however did not meet our purpose of testing standard profilometric catheters.

Multi-channel measurements with vector graphic analysis were used ${ }^{8,9}$ for the evaluation of profilometric parameters of incontinent female patients, and for testing of reproductibility ${ }^{10}$, also with satisfactory experience.

\section{CONCLUSION}

Regarding the fact that the examinations were easy reproducible even after the entire cycle of measurements, we consider bull urethra to be sufficiently stable and resistant for use as a test model for urethra profilometry.

Software equipment with the possibility of free programming of measuring protocols provided to connect a wide range of measuring catheters and top models including new models for which yet no standard apparatus has been available.

The evaluation of particular measurements using the superposition of pressure curves obtained from various positions of a catheter in the urethra, as well as a display of multi-channel measurement through vector graphs, allowed a thorough study of this issue.

\section{ACKNOWLEDGEMENTS}

The preparation of this work was supported by an IGA grant Nr 7840-3 /5211036 and GACR grant, Nr 106/04/ 020150110111

\section{REFERENCES}

1. Brown M, Wickham JEA. The urethral pressure profile. Br J Urol 1969; 41:211-7.

2. Griffiths D. The pressure within a collapsed tube, with special reference to urethral pressure. Phys Med Biol 1985; 30:951-63.

3. Harrison NW, Constable AR. Urethral Pressure Measurement: a modified Technique. BJU 1970; 42:229-233.

4. Abrams PH, Martin S, Griffiths DJ. (1977) The Measurements and Interpretations of urethral pressures by the Method of Brown and Wickham. Br J Urol (1978) 150:33-8.

5. Lose G, Griffiths D, Hosker D, Kulseng-Hanssen S, Perucchini D, Schäfer W, Thind P, Versi E. Standardisation of Urethral Pressure Meassurement:Report from the Standardisation Sub-committee of the International Continence Society. Neurourol. Urodyn Issue 2, 2002.

6. Gilmour R.F, James D.F, Toguri A.G, Churchill B.M. Analysis of the Urethral pressure profile using a mechanical Model. Jurol 1980; 18, 1:54-58.

7. Rawlings CA, Coates JR, Chernosky A, Barsanti JA, Oliver JE. Stress leak point pressures and urethral pressure profile test in clinical normal female dogs. Am J Vet Res Jun, 1999; 60, 6:676-8.

8. Haeusler G, Temper AE., Heinzl A, SAM CH, Hefler L, Hanzal E, Keoelbl H. Value of Urethral Pressure Profilometry in the Female Incontinent Patient: A Prospective Trial With an 8-Channel Urethral Catheter. Urology 1998; 52, 6:1113-1118.

9. Messelink MD, Dabhoiwala, NB,Vrij V. et al. Multichannel urethral pressure profiles:reproductibility and three-dimensional representation. J.Urol 1997; 11, 3:211-214. 